\title{
Central retinal artery occlusion (CRAO), Is it a form of stroke?
}

Miloni Shah $^{1{ }^{1 *},}$ Kalpana Gadsingh ${ }^{1}$, and Pranjali Pawar ${ }^{1}$

\author{
${ }^{1}$ Dr. Vasantrao Pawar Medical College Hospital and Research Centre, Adgaon, Nashik 422207, India
}

\begin{abstract}
Central retinal artery occlusion (CRAO) is an ophthalmic emergency. It occurs when central retinal artery gets occluded. We present a case of a 34-years-old male, alcoholic and a smoker presenting with sudden loss of vision of right eye. Fundus examination revealed a picture of CRAO. On further neuroimaging, a silent acute to subacute lacunar infarcts were found.
\end{abstract}

Keywords: Central retinal artery occlusion; stroke; lacunar infarcts

*Corresponding author: Dr. Miloni Shah, Dr. Vasantrao Pawar Medical College Hospital and Research Centre, Adgaon, Nashik 422207, India. Mobile: +919920699445; Email: miloni445@ gmail.com

Received 11 April 2019; Revised 31 May 2019; Accepted 19 June 2019; Published 27 June 2019

Citation: Shah M, Gadsingh K, Pawar P. Central retinal artery occlusion (CRAO), Is it a form of stroke? J Med Sci Res. 2019; 7(3):90-92. DOI: http://dx.doi.org/10.17727/JMSR.2019/7-15

Copyright: (C) 2019 Shah M et al. Published by KIMS Foundation and Research Center. This is an open-access article distributed under the terms of the Creative Commons Attribution License, which permits unrestricted use, distribution, and reproduction in any medium, provided the original author and source are credited.

\section{Introduction}

The retina receives its blood supply from two sources. The choriocapillaris of the choroid supplies the outer retina, whereas the central retinal artery (CRA) and its end artery branches feed the inner retinal layers. These circulations both originate from the ophthalmic artery, the CRA being its first branch and the ciliary arteries giving rise to the choriocapillaris. Occasionally, a cilioretinal artery arises from the ciliary circulation and supplies a portion of the papillomacular bundle. Central retinal artery occlusion occurs when the central retinal artery gets blocked, usually due to an embolus or a thrombus. The retinal artery occlusion may be transient and last for only a few seconds or minutes if the blockage breaks up and restores blood flow to the retina, or it may be permanent. It causes sudden, painless, unilateral, and usually severe vision loss. Diagnosis is by history and characteristic retinal findings on fundoscopy.

\section{Case report}

A 34-years-old male presented to us with a history of sudden painless loss of vision of right eye of 6 days duration. The loss of vision was also associated with 
headache of duration of 6 days. There was no history of trauma, systemic illness. Patient also gives history of alcohol intake twice or thrice a week (120-160 $\mathrm{ml}$ each time). Patient was also a smoker. Patient's visual acuity at presentation was OD No PL OS was $6 / 6$ with presence of relative afferent pupillary defect (RAPD) in OD. Rest of the anterior segment examination was normal. Fundus examination OD revealed optic disc odema and pallor with cherry red spot at the macula and a pale posterior pole with arterial attenuation and superficial haemorrhages in periphery. Extraocular moments were normal (Figure 1).

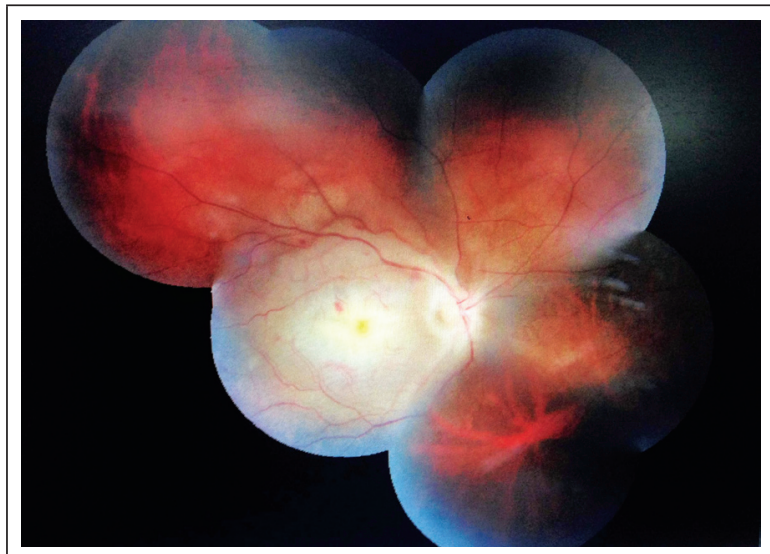

Figure 1: Fundus photo showing central retinal artery occlusion.

On systemic examination blood pressure was $128 / 84 \mathrm{mmHg}$. Routine blood investigations along with erythrocyte sedimentation rate (ESR) and c-reactive protein (CRP) was done to rule out giant cell arteritis. Coagulation profile studies which included anti cardiolipin antibodies, protein C, protein S, ANA and serum homocysteine along with lipid profile were also done and all the test results were within normal range.

MRI brain revealed multiple acute to subacute lacunar infarcts in right Fronto- Parietal cortical and subcortical region, right Ganglio-Thalamo-capsular region and right periventricular white matter. Absent flow void was noted in right ICA with FLAIR hyper intensive signal noted within its lumen suggestive of an occlusion (Figure 2).

After consultation with the neurologist and cardiologist other medical management was started with dual antiplatelet therapy with tablet aspirin and clopidogrel 75/75 mg. The patient was stable and followed up regularly with no signs and symptoms of further systemic stroke.

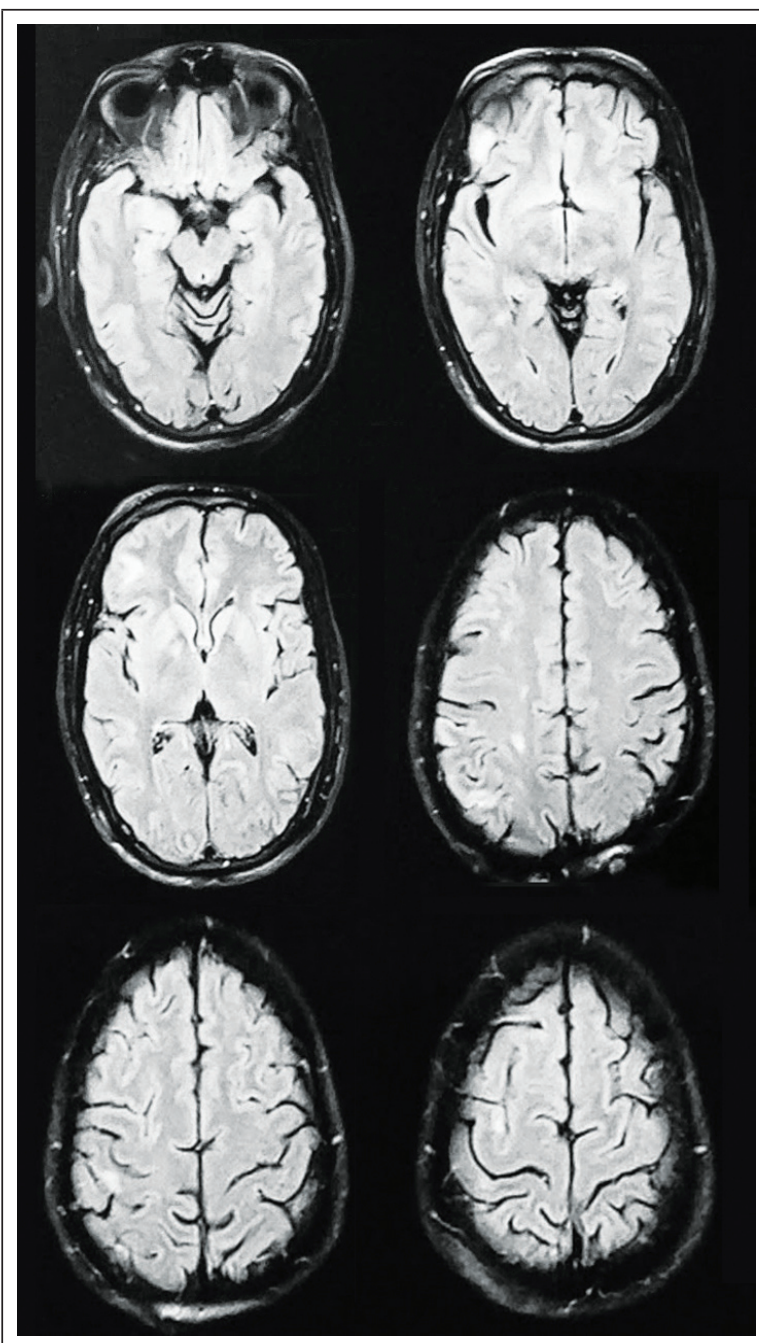

Figure 2: MRI report showing multiple lacunar infarcts in right Fronto-Parietal cortical and subcortical region, right Ganglio- Thalmo capsular region and right periventricular white matter

\section{Discussion}

The central retinal artery enters the globe at the optic disc where it bifurcates into superior and inferior branches, each of which in turn bifurcates into nasal and temporal branch retinal arteries. Therefore, occlusion of the central retinal artery deprives the entire inner retina of its blood supply unless a cilioretinal artery is present $(15-30 \%$ of eyes) [1]. Arterial occlusive disease of the retina is the result of either arteriosclerotic thrombosis, vasculitis, embolic impaction, vasospasm or systemic hypotension. 
CRAO per se has no standard treatment. In Indian scenario by the time patient approaches an ophthalmologist it is already too late to salvage the vision. Various methods non invasive and invasive have been tried. Non invasive methods include giving a trial of ocular massage, hyperbaric oxygen, anterior chamber paracentesis, medical management to reduce intraocular pressure (IOP). Invasive method includes intra-arterial fibrinolysis. Ahn et al. showed that approximately $40 \%$ of patients undergoing intra-arterial thrombolysis showed a no-reflow phenomenon, and those with the no-reflow phenomenon suffered a worse visual outcome, with more retinal atrophy and disruption of photoreceptors [2]. However, in a separate study, Ahn et al. concluded that intra-arterial thrombolysis may help patients with incomplete CRAO [3].

The European Assessment Group for Lysis in the Eye (EAGLE) [4] compared the effect of intraarterial tPA to "conservative" treatments (e.g., IOP lowering medications, IV heparin, hemodilution, ocular massage, daily ASA therapy). There was no statistically significant improvement in visual acuity after intra-arterial tPA administration compared to conservative treatment. The study was terminated at the first interim analysis because of the increased incidence of adverse events in the tPA treatment group.

CRAO has to be treated as a medical emergency! The incident rate ratio for ischemic stroke peaks 1 to 7 days after CRAO (44.51; 95\% CI, 27.07-73.20) and remains elevated for the first 30 days $(14.0 ; 95 \% \mathrm{CI}$, 8.90-22.00) [5].

A study [6] suggested $36.7 \%$ of patients had critical carotid disease, $37.3 \%$ had coincident acute stroke, $33.0 \%$ presented with hypertensive emergency, $20.0 \%$ had a myocardial infarction or critical structural cardiac disease, $25 \%$ underwent an urgent surgical intervention, and $93 \%$ had a change in medication as a result of the inpatient evaluation. Patients with CRAO had similar risk of subsequent stroke, myocardial infarction, and death as patients with high-risk transient ischemic attack.

\section{Conclusion}

CRAO per se might not have a good prognosis but it can definitely prevent risk of future strokes by identifying and treating the patients at risk. Silent brain infarction may be a surprise finding in a case of CRAO. Hence it is an absolute necessary to subject each patient of CRAO to an urgent extensive investigation and a promptreference to a cardiologist, neurologist combined with neuro-ophthalmologist to treat the patient and to keep a follow in order to be alert about the sequale which follow it.

\section{Declaration of patient consent}

The authors certify that they have obtained all appropriate patient consent forms. In the form the patient(s) has / have given his/ her/ their consent for his/ her/ their images and other clinical information to be reported to the journal. The patient understands that their names and initial will not be published and due efforts will be made to conceal their identity, but anonymity cannot be guaranteed.

\section{Conflicts of interest}

Authors declare no conflicts of interest.

\section{References}

[1] Hegde V, Deokule S, Matthews T. A case of a cilioretinal artery supplying the entire retina. Clin Anat. 2006; 19(7):645647.

[2] Pielen A, Pantenburg S, Schmoor C, Schumacher M, Feltgen $\mathrm{N}$, et al. Predictors of prognosis and treatment outcome in central retinal artery occlusion: local intra-arterial fibrinolysis vs. conservative treatment. Neuroradiology. 2015; 57(10):1055-1062.

[3] Ahn SJ, Park KH, Ryoo NK, Hong JH, Jung C, et al. No-reflow phenomenon in central retinal artery occlusion: incidence, risk factors, and clinical implications. PLoS One. 2015; 10(11):e0142852.

[4] Page PS, Khattar NK, White AC, Cambon AC, Brock GN, et al. Intra-arterial thrombolysis for acute central retinal artery occlusion: a systematic review and meta-analysis. Front Neurol. 2018; 9:76.

[5] Park SJ, Choi NK, Yang BR, Park KH, Lee J, et al. Risk and risk periods for stroke and acute myocardial infarction in patients with central retinal artery occlusion. Ophthalmology. 2015; 122(11):2336-2343.

[6] Lavin P, Patrylo M, Hollar M, Espaillat KB2, Kirshner H, et al. Stroke risk and risk factors in patients with central retinal artery occlusion. Am J Ophthalmol. 2018; 196:96-100. 